FOUNDATIONS

ADVANCES

\title{
Cryo-coherent diffractive imaging of biological samples with X-ray free-electron lasers
}

\author{
Huaidong Jiang* \\ State Key Laboratory of Crystal Materials, Shandong University, Jinan 250100, People's Republic of China. \\ *Correspondence e-mail: hdjiang@sdu.edu.cn
}

Received 16 February 2016 Accepted 22 February 2016

Keywords: X-ray free-electron lasers; cryocoherent diffractive imaging; CDI; XFELs; biological specimens.

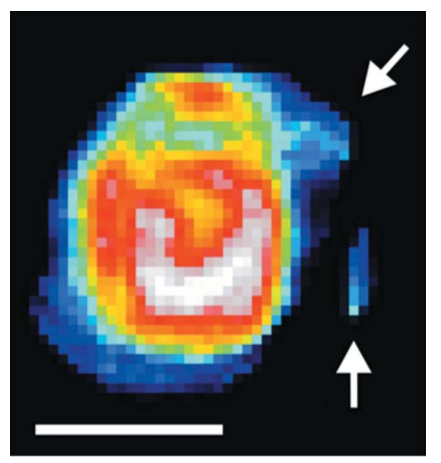

C 2016 International Union of Crystallography
As the functions of living organisms are closely related to their structures, high-resolution imaging in the natural state is a long-term goal for researchers. To achieve this goal, two important things are needed: (1) a high-resolution imaging method and (2) an effective method for preparing biological samples that keeps them in their natural state. In this issue, Takayama \& Yonekura (2016) report the progress and achievements in cryogenic coherent X-ray diffractive imaging of biological samples such as cells and organelles at the SPring-8 Angstrom Compact free-electron LAser (SACLA). Although cell imaging has been realized before with single-shot coherent diffractive imaging (CDI) (Kimura et al., 2014; van der Schot et al., 2015), the combination of cryogenic techniques and radiation-damage-free single-shot CDI offers the potential for quantitative imaging of biological samples in their natural state with high spatial resolution. This study is encouraging and has important implications for a better understanding of the structures of cells at a particular period of the cell cycle or in a particular environment.

In contrast to conventional imaging methods, CDI (Miao et al., 1999) is a lensless technique. It has emerged as a powerful method for in situ high-resolution imaging as it takes advantage of the long penetration length of X-rays and circumvents the barriers associated with lens-based imaging (Miao et al., 2015). Most of the initial CDI experiments were conducted using third-generation synchrotron-radiation facilities. In recent years, a novel and powerful radiation source, the X-ray free electron laser (XFEL), has been developing rapidly and many are now being constructing worldwide. CDI using an XFEL takes advantage of the nearly full spatial coherence, the ultra-short pulse duration and the ultra-brightness of the radiation, and is highly suited to imaging cells without causing radiation damage.

Chemical fixation and freeze-drying are the two methods that are widely used for the preparation of biological samples for X-ray imaging (Jiang et al., 2010; Nelson et al., 2010; Nishino et al., 2009). However, artificial processing is inevitable in these methods and it affects the natural structures. For instance, living cells are very sensitive to environmental changes, such as pressure and temperature. The frozen-hydration technique is one of the best choices, as it keeps biological samples as close as possible to their living conditions. Cryogenic coherent diffractive imaging (cryo-CDI) using synchrotron radiation has been used for both two-dimensional (Huang et al., 2009; Lima et al., 2009) and threedimensional (Rodriguez et al., 2015) imaging of single cells.

Advances in imaging techniques have also provided more convenient ways to achieve high-resolution images. Takayama and Yonekura (Takayama \& Yonekura, 2016; Takayama et al., 2015) report an effective strategy for combining frozen-hydrated sample preparation with XFEL-CDI while maintaining the hydrated state of the sample, using a special experimental setup at the SACLA XFEL light source. Additionally, specific control and data-processing software [the G-SITENNO suite (Sekiguchi, Oroguchi et al., 2014; Sekiguchi, Yamamoto et al., 2014)] has been developed, which enables automatic background subtraction, merging of high- and low-resolution diffraction patterns, and display of the diffraction patterns.

One of the advantages of XFEL-CDI is that it offers 'diffraction before sample destruction', which enables one to image living cells (van der Schot et al., 2015). A microliquid enclosure (Kimura et al., 2014) or an aerosol sample injector (van der Schot et al., 2015) are usually used for live cell imaging. The use of cryo-XFEL-CDI to image frozenhydrated cells avoids adverse environmental affects that might arise during sample preparation, so ultimately provides the true morphology and inner structures of the cells. 
(a)

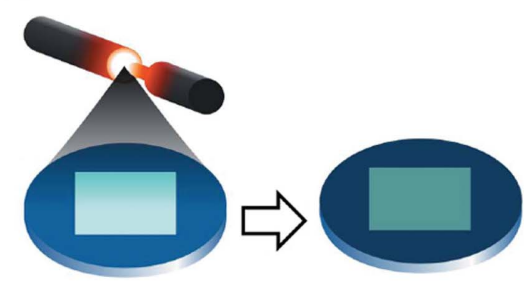

Sample support disk

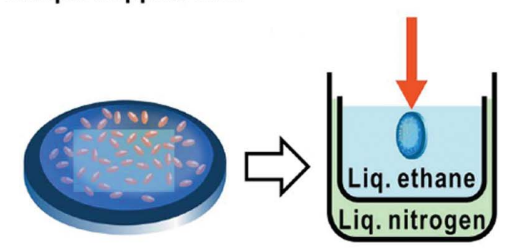

(b)

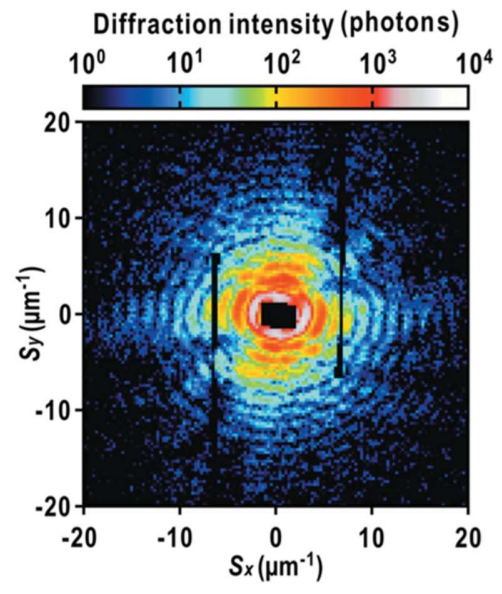

(c) Projected electron density (a.u)

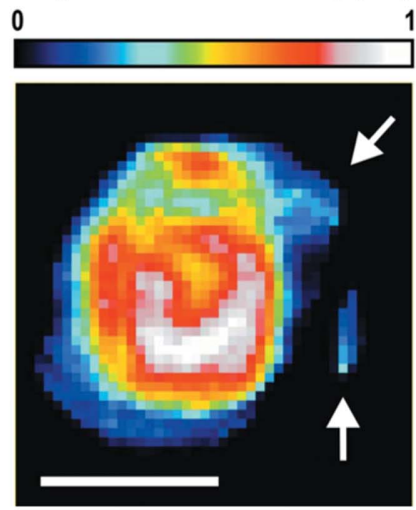

Figure 1

Outline of a cryo-XFEL-CDI experiment on a biological sample. (a) A schematic diagram of the stepwise preparation of frozen-hydrated biological samples on the support membrane. (b) A diffraction pattern from a frozen-hydrated minicell. ( $c$ ) The corresponding reconstructed electron-density map of the minicell from the diffraction pattern in $(b)$. Scale bar: $500 \mathrm{~nm}$. [Reproduced with permission from Takayama \& Yonekura (2016).]

One key issue for cryo-XFEL-CDI is preparing suitable flashfrozen samples. Both the ice thickness and the density of the cells on the membrane should be controlled to lie within a reasonable range. In Takayama and Yonekura's article, optimized conditions have been determined for obtaining flashcooled samples with suitable ice thickness and sample density by keeping the relative humidity at around $100 \%$ and using carbon membranes or silicon-nitride membranes coated with carbon. Fig. 1(a) shows the sample-preparation scheme that they used. A cryogenic electron microscope (EM) or light microscope (LM) was used to check the results. The combination of cryo-XFEL-CDI with cryo-EM or LM provided fast feedback about whether the samples had been prepared at optimized conditions. The density of isolated samples on the membranes was an important factor in maximizing the interaction of the sample with the XFEL beam.

Takayama \& Yonekura used their new method to study chloroplasts isolated from C. melorae and minicells grown from Escherichia coli strain ME8077. Fig. 1(b) shows a typical diffraction pattern obtained from a frozen-hydrated minicell. A reconstructed electron-density map with an effective resolution of $52 \mathrm{~nm}$, obtained using the HIO-SW and OSS algorithms, is shown in Fig. 1(c). Despite the use of ultra-bright XFEL radiation, the resolution achieved in the reconstructions of biological particles is still limited because of the small scattering cross section. However, when using colloidal gold particles as a reference, the total diffraction signal clearly increased. The introduction of colloidal gold particles meant that the Patterson-search phasing method could be used for phase retrieval and reconstruction. Takayama and Yonekura's impressive study not only demonstrates cryo-XFEL-CDI of biological samples in their natural state, but also describes a useful diffraction-enhancement method together with robust phase retrieval.

\section{References}

Huang, X., Nelson, J., Kirz, J., Lima, E., Marchesini, S., Miao, H., Neiman, A. M., Shapiro, D., Steinbrener, J., Stewart, A., Turner, J. J. \& Jacobsen, C. (2009). Phys. Rev. Lett. 103, 198101.

Jiang, H., Song, C., Chen, C. C., Xu, R., Raines, K. S., Fahimian, B. P., Lu, C., Lee, T., Nakashima, A., Urano, J., Ishikawa, T., Tamanoi, F. \& Miao, J. (2010). Proc. Natl Acad. Sci. USA, 107, 11234-11239.

Kimura, T., Joti, Y., Shibuya, A., Song, C., Kim, S., Tono, K., Yabashi, M., Tamakoshi, M., Moriya, T., Oshima, T., Ishikawa, T., Bessho, Y. \& Nishino, Y. (2014). Nat. Commun. 5, 3052.

Lima, E., Wiegart, L., Pernot, P., Howells, M., Timmins, J., Zontone, F. \& Madsen, A. (2009). Phys. Rev. Lett. 103, 198102.

Miao, J., Charalambous, P., Kirz, J. \& Sayre, D. (1999). Nature, 400, 342-344.

Miao, J., Ishikawa, T., Robinson, I. K. \& Murnane, M. M. (2015). Science, 348, 530-535.

Nelson, J., Huang, X., Steinbrener, J., Shapiro, D., Kirz, J., Marchesini, S., Neiman, A. M., Turner, J. J. \& Jacobsen, C. (2010). Proc. Natl Acad. Sci. USA, 107, 7235-7239.

Nishino, Y., Takahashi, Y., Imamoto, N., Ishikawa, T. \& Maeshima, K. (2009). Phys. Rev. Lett. 102, 018101.

Rodriguez, J. A., et al. (2015). IUCrJ, 2, 575-583.

Schot, G. van der, et al. (2015). Nat. Commun. 6, 5704.

Sekiguchi, Y., Oroguchi, T., Takayama, Y. \& Nakasako, M. (2014). J. Synchrotron Rad. 21, 600-612.

Sekiguchi, Y., Yamamoto, M., Oroguchi, T., Takayama, Y., Suzuki, S. \& Nakasako, M. (2014). J. Synchrotron Rad. 21, 1378-1383.

Takayama, Y. \& Yonekura, K. (2016). Acta Cryst. A72, 179-189.

Takayama, Y., Yonekura, S. M., Oroguchi, T., Nakasako, M. \& Yonekura, K. (2015). Sci. Rep. 5, 8074. 